\title{
Arthroscopic Rotator Cuff Repair: Advancements in Techniques and Technology
}

\author{
Claudio Chillemi, $M D^{1^{*}}$, Mario Guerrisi, $M D^{2}$, Giuseppe Polizzotti, MD², Carlo Paglialunga, \\ $M D^{2}$, Stefano Carli, $M D^{2}$ and Marco Damo, $M D^{2}$
}

${ }^{1}$ Department of Orthopaedic Surgery, Istituto Chirurgico Ortopedico Traumatologico, Italy

${ }^{2}$ Department of Anatomical, Histological, Forensic Medicine and Orthopaedics Sciences, Sapienza University of Rome, Istituto Chirurgico Ortopedico Traumatologico, Italy

\begin{abstract}
The surgical treatment of rotator cuff tear, thanking to the advancement in technology and technique, has evolved during time. The tendon healing process is strictly correlated to the existing techniques of suturing, and to biological and mechanical factor.

The aim of this paper is to update the advancements in techniques and technology for rotator cuff repair and propose an algorithm for rotator cuff fixation based on tissue quality and tear configuration.

In conclusion, now a days, is possible to affirm that doesn't exist anymore an irreparable rotator cuff tear, but exist rotator cuff tear that are unable to heal. In the next future the main topic of the research has to be the improvement of the biology.
\end{abstract}

Keywords

Rotator cuff, Tear, Healing, Anchors, Single row, Double row, Transosseous equivalent

\section{Introduction}

The successful management of a symptomatic rotator cuff tear is represented by surgery, reattaching the tendon to bone at the original site of insertion. In spite of the significant improvement in the surgical approach, the rate of re-tear is still high and seems to be related to biological and mechanical factor, and the existing techniques of suturing [1].

Different attempts were described in literature with the aim to improve the biology of the tendon healing process. On the other hands, thanking to the advancements in techniques and technology, the biomechanics of the repair was improved, restoring the anatomy as closely as possible so to achieve high initial fixation strength, minimizing gap formation, and maximizing the contact area [2].

A wider and more stable tissue-bone contact during the early phase of fibrillogenesis in tendon healing is a key concept, and for this reason a smaller contact area and pressure have a potential risk for higher structural failure rates [3].

The knowledge of the biomechanics of the repair bring the conclusion that traditional transosseous (TO) techniques [4] were superior to single-row (SR) techniques in ultimate load to failure, interface motion, restoring the footprint, and achieving the best pressure on the repair [5]. Until a few years ago, rotator cuff to repair technique was considered the gold standard procedure [6]. With the advent of arthroscopy the approach has changed and numerous arthroscopic techniques have been described [7]. Trying to reproduce arthroscopically the classic TO approach with anchors, double row (DR) and Transosseous-Equivalent (TOE) techniques were developed [8].

In particular, the latter has been proved to be a valid solution taking into account the biomechanical aspects, with reduced gap formation and self-reinforcement capability (i.e. the ability to create a simultaneous resistive frictional force over a repaired footprint) as a distractive and potentially destructive tendon load is applied. At the same time as surgical techniques, materials have also undergone development over

*Corresponding author: Claudio Chillemi, MD, Department of Orthopaedic Surgery, Istituto Chirurgico Ortopedico Traumatologico (I.C.O.T.), Latina, Italy, Tel: +39-0773-6513213, Fax: +390773-418261

Accepted: March 23, 2021

Published online: March 25, 2021

Citation: Chillemi C, Guerrisi M, Polizzotti G, et al. (2021) Arthroscopic Rotator Cuff Repair: Advancements in Techniques and Technology. Adv Tech Musculoskelet Surg 5(1):59-65 
Citation: Chillemi C, Guerrisi M, Polizzotti G, et al. (2021) Arthroscopic Rotator Cuff Repair: Advancements in Techniques and Technology. Adv Tech Musculoskelet Surg 5(1):59-65

Table 1: Evolution during time of the anchors materials.

\begin{tabular}{|c|c|c|}
\hline Material & Features & IN VIVO Reaction \\
\hline METAL & $\begin{array}{l}\text { - } \quad \text { Easy to assess changes on conventional X-ray } \\
\text { - Greater pull-out strength }\end{array}$ & $\begin{array}{l}\text { High biocompatibility; } \\
\text { Difficult in revision case } \\
\text { Interference with MRI }\end{array}$ \\
\hline PGA & $\begin{array}{ll}\text { - } & \text { Bioabsorbable } \\
\text { - } & \text { Rapid resorption }\end{array}$ & $\begin{array}{l}\text { Hydrolysis of the polymer, cleared by local } \\
\text { macrophages which resulted in lytic bone changes }\end{array}$ \\
\hline PLLA & $\begin{array}{l}\text { - } \quad \text { Bio absorbable } \\
\text { - Slow resorption (up to } 5 \text { years) }\end{array}$ & $\begin{array}{l}\text { Change in molecular weight and crystallinity, } \\
\text { reduction mechanical properties of the anchor and } \\
\text { failure due to breakdown }\end{array}$ \\
\hline TCP & $\begin{array}{l}\text { - } \quad \text { Bio absorbable } \\
\text { - } \quad \text { Mineral content similar to the natural bone }\end{array}$ & $\begin{array}{l}\text { Macroporosity and microporosity conducive to } \\
\text { osteoconductivity, compressive strength, } \\
\text { dissolution, and biocompatibility }\end{array}$ \\
\hline PEEK & $\begin{array}{ll}\text { - } & \text { Non-biodegradable } \\
\text { - } & \text { Resistant to chemical, thermal and radiant agents }\end{array}$ & $\begin{array}{l}\text { Inert; High strength, radiolucent can be drilled out in } \\
\text { revision cases }\end{array}$ \\
\hline ALL-SUTURE & $\begin{array}{l}\text { - Soft } \\
\text { - } \quad \text { A mall size } \\
\text { the pilot hole } \\
\text { - Reducing the risk of pullout }\end{array}$ & $\begin{array}{l}\text { Reduced risk of adverse reactions; non interference with } \\
\text { MRI; less complex revisions }\end{array}$ \\
\hline
\end{tabular}

PGA : Polyglycolic Acid, PLLA: Polylactic Acid, TCP: Tricalcium Phosphate, PEEK: Polyetheretherketone

Table 2: List of The Anchors Described in This Study To Perform a Transosseous Equivalent Rotator Cuff Repair.

\begin{tabular}{|l|l|l|l|}
\hline Commercial name & Dimension $\mathbf{( m m )}$ & Types of suture & Tape \\
\hline Medial row & & Cord-like & \\
\hline Iconix 2 & & & \\
\hline Iconix 3 & 2.3 & 2 strands \#2 force fiber & \\
\hline Iconix 2 TT & 2.3 & 3 strands \#2 force fiber & 2 strands Xbraid TT (2.0 mm) \\
\hline Iconix Speed Combo & 2.3 & & 2 strands Xbraid TT (1.2 mm and 2.0 mm) \\
\hline Iconix Speed double loaded & 2.3 & & 2 strands Xbraid TT (2.0mm) \\
\hline Iconix Speed double loaded & 2.3 & 2 strands \#2 force fiber & \\
\hline Iconix Speed triple loaded & 2.3 & & 3 strands Xbraid TT (1.2 mm) \\
\hline & & & \\
\hline Lateral row & & Knotless Anchor & \\
\hline ReelX STT & 4.5 & & \\
\hline
\end{tabular}

The Speed family includes all self-punching anchors.

the years, in particular the fixation devices, such as type of anchors (Table 1) and sutures. In addition to the cord-like conventional wires, the tape (flatter and wider) was introduced into the market with the rationale to maximize the pressure and the tendon-bone contact area so to promote healing [9].

The aim of this paper is to update the advancements in techniques and technology for rotator cuff repair and propose an algorithm for rotator cuff fixation based on tissue quality and tear configuration.

\section{Materials and Methods}

Before starting the surgery, some information appears mandatory, regarding at first the patient and the tear. It ap- pears essential the knowledge of 1) The onset of symptoms (i.e. acute or chronic that means traumatic or degenerative origin of the tear) 2) The type of tear and tissue quality. Obviously the imaging is crucial to clarify this aspect. X-ray is helpful to evaluate the gleno-humeral joint and the bone quality, while the tear pattern, the extent of retraction and soft tissue quality are better evaluated by MR [10]. These aspects are all considered in different classification system, and included into the system proposed by ISAKOS Upper Limb and Arthroscopy Committees. This classification encompasses five essential characteristics with regard to tears - pattern $(P)$, extension $(E)$, fatty atrophy $(A)$, retraction $(R)$, and location $(L)$ - conforming the acronym "PEARL" 
[11]. The ISAKOS group recommended to follow the classification system of full-thickness postero-superior rotator cuff tears suggested by Snyder [12] that provides information not only on the size but also on the number of tendons involved and the degree of retraction and scarring of the remaining tendon so providing information on the repair ability of the lesion.

Another aspect to be considered is the material to be employed for the repair. In case of DR or TOE repair the surgeon have to choose both anchors for the medial and lateral row that may be solid or all-soft (Table 2). Medial row: "All-soft" suture anchors (authors preference) are composed entirely of suture. These anchors have standard high-strength suture limbs (cord-like or tape) that are attached to a stiff suture section that is placed into a bone socket, and they deploy with tension on the suture limbs to achieve subcortical fixation. The technology adopted creates a bunching effect using targeted compression zones within the implant sheath for optimal fixation. The anchor design help to minimize bone removal and it requires a smaller bone socket than a solid anchor [3]. These advantages are reinforced by a biomechanical performance that is similar to the classic solid anchor [13].

\section{Lateral row}

For this row may be usually used solid anchors. The knotless anchor (author preference) is a device that uses a new concept for tissue fixation, that simplifies the well known knot fixation used by traditional anchors. The knotless anchor allows easy and secure suture fixation to the bone with an interferential mechanism, without knots. These improvements facilitating the performance of arthroscopic shoulder surgery reduce operative time.

The preferred knotless anchor device has a metal tip that allows it to be inserted directly into the tuberosity by tapping it. On the body of the anchor there are 2 eyelets inside which up to four cord-like sutures \#2 can be loaded. PEEK anchor body expands up to one full millimetre in diameter under the cortical surface and with the incremental tension system, patient Specific Tissue Tension and greater control over repair is possible. This device has a suture winding mechanism to maximize the contact area: The implant has one locking point for every $60^{\circ}$ of revolution of the knob, and advances approximately $1.5 \mathrm{~mm}$ of suture for every $60^{\circ}$ of rotation.

\section{Surgical technique}

The procedure can be performed depending on anaesthesiologist preference under general anaesthesia or interscalene cervical plexus block [14] or combined, and in beachchair position or lateral decubitus according with surgeon request. A three-portal surgical technique is suggested: Posterior (for the scope), anterior and lateral (working) portals. A percutaneous small accessory supero-lateral portal is required for the anchor insertion. The main technical principles to be adopted in every case are below reported.

\section{Greater tuberosity preparation}

A large tuberosity preparation is obtained with cruentation of the bone surface laterally with respect to the area in which the medial anchors are positioned; this last aspect is important in order to avoid weakening the bone that may lead to the pull-out of the anchors. Microperforations are also performed to create an optimal environment for biological healing by exploiting the bone marrow growth factors, platelets and mesenchymal stem cells [15].

\section{Tendon preparation}

Bleeding of the lesional stump of the tendon is performed in order to remove the fibrous component which is not functional for repair, in particular in chronic lesions where often there is a margins fibrocartilaginous metaplasia of the torn tendon [16]. Lysis from adhesions is performed to reduce retraction and allow the tendon to reach the footprint.

\section{Anchor positioning}

Through a percutaneous small accessory supero-lateral portal, medial row anchors are placed near the cartilage margin to avoid the pullout of the anchor.

For the lateral row knotless anchors are used through lateral working portal: Based on the type of lesion and its mobility, the anchors are positioned as distal as possible to the apex of the greater tuberosity, where a higher pullout strength has been demonstrated [17]. When 2 lateral anchors are used is advisable to maintain an 8-10 $\mathrm{mm}$ "bone bridge" between them.

Depending on tear size, different configurations can be performed in order to obtain the construct that is considered biomechanically suitable.

\section{Small Tear (Snyder C1)}

\section{Configuration \#1}

Medial row: The construct is made according to the following method: One soft anchor double loaded is placed medially whose limbs are passed through the tendon from posterior to anterior and proceed to close the medial row with mattress nodes in the opposite way. Lateral row. Each pair of threads is loaded on a knotless anchor (ReelX STT) and placed laterally in order to obtain a suture bridge-like configuration (Figure 1).

\section{Configuration \#2}

Medial row: Same construct of configuration \#1.

Lateral row: A thread from each pair of wire, of different color, is loaded on a knotless anchor and then tensioned in order to crush the tendon against the bone. The final configuration will be a double row $\mathrm{X}$-box (Figure 2 ).

\section{Configuration \#3}

Medial row: A self-punch anchor (ICONIX Speed Combo) with 2 tapes of different sizes $(2 \mathrm{~mm}$ and $1.2 \mathrm{~mm}$ ) is employed. All tapes are passed through the tendon with the largest one positioned respectively more posterior and more anterior, while the smaller are in the center. The smaller tape is closed with mattress knot and maintained.

Lateral row: Each pair, of different tape sizes, is inserted 


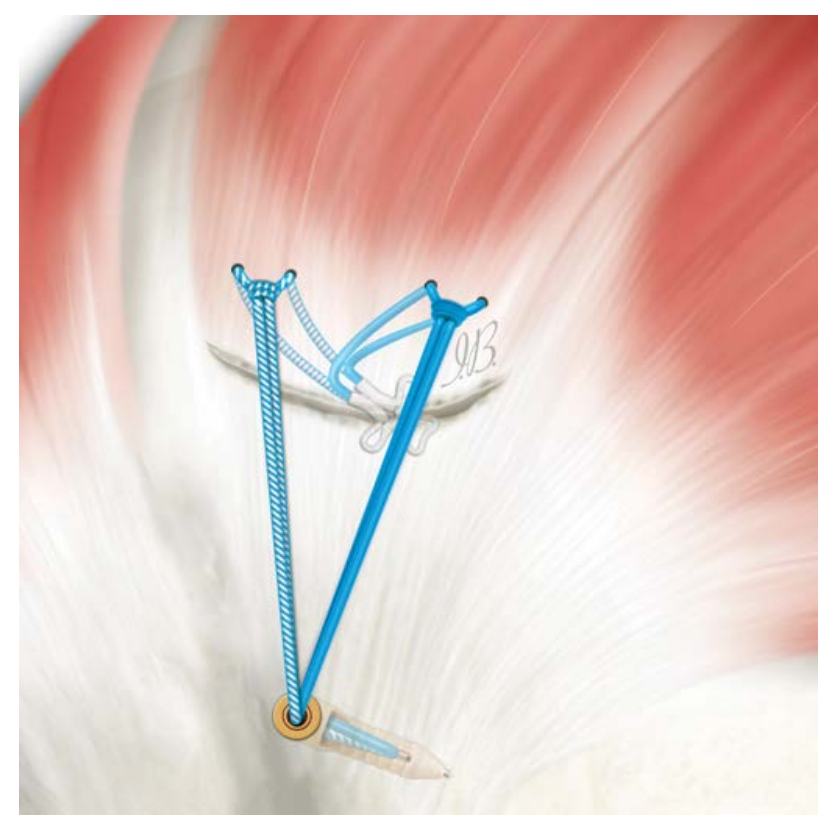

Figure 1: Configuration \#1.

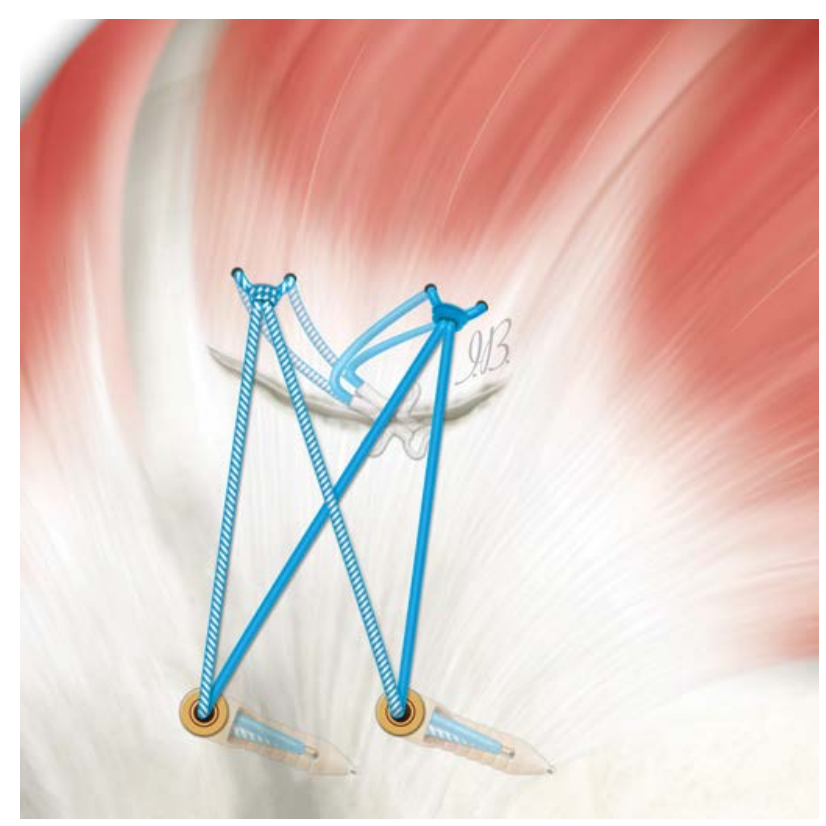

Figure 2: Configuration \#2.

into the eyelets of a knotless anchor which is positioned laterally (Figure 3a).

Configuration \#3.1: In the same way, a similar configuration can be obtained by cutting the central tape once knotted (Figure 3b).

Configuration \#3.2: Similarly two lateral anchors can be positioned to obtain greater coverage of the footprint and reduce tension (Figure 3c).

\section{Moderate Tear (Snyder C2)}

\section{Configuration \#4}

Medial row: Each wire of different colours of a soft an-
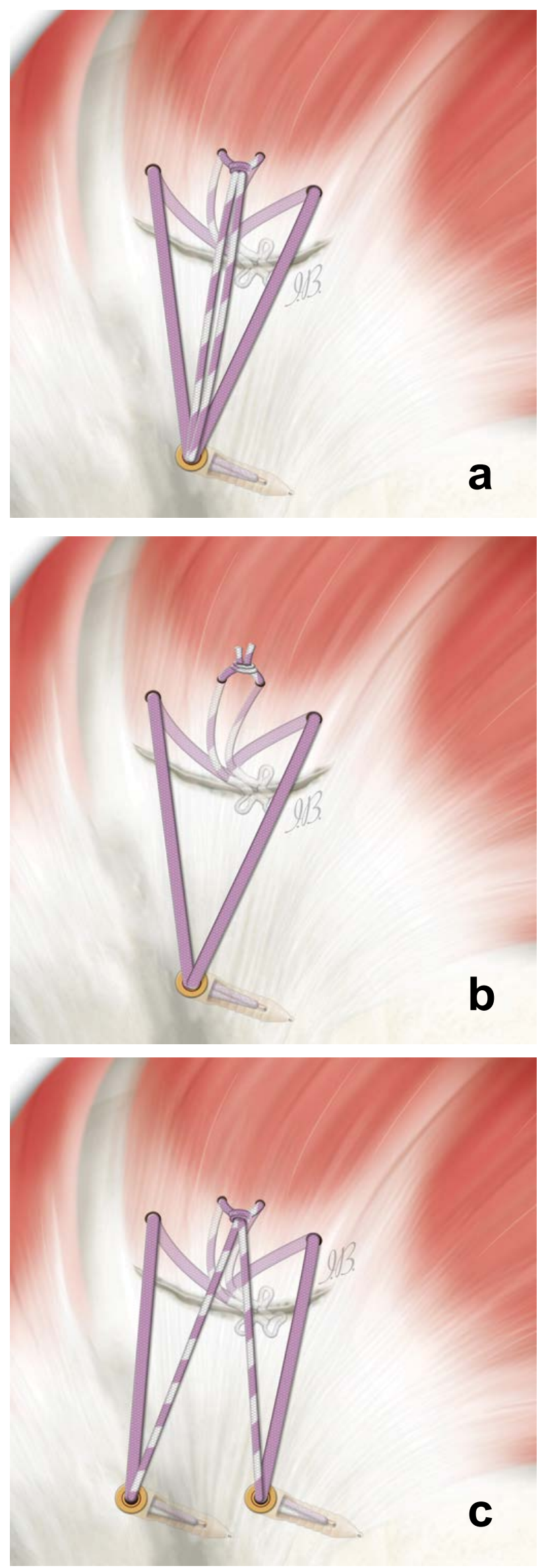

Figure 3: Configuration \#3. 
chor triple loaded with cord-like wires (Iconix 3) or tape (Iconix Speed Triple Loaded) is passed through the tendon from the posterior to the anterior. The first row is tightened in an anterior-posterior direction with mattress knot.

The suture tails are grouped in pairs of 3 , based on color, and each pair is then loaded on the 2 knotless anchors which are positioned laterally (Figure $4 a$ ).

\section{Configuration \#4.1}

The tapes/wires of the central knot is cut and the remaining pair of sutures are used as in the construct described above (Figure 4b).

\section{Large Tear (Snyder C3)}

\section{Configuration \#5}

Medial row: This construct involves the use of 2 medial
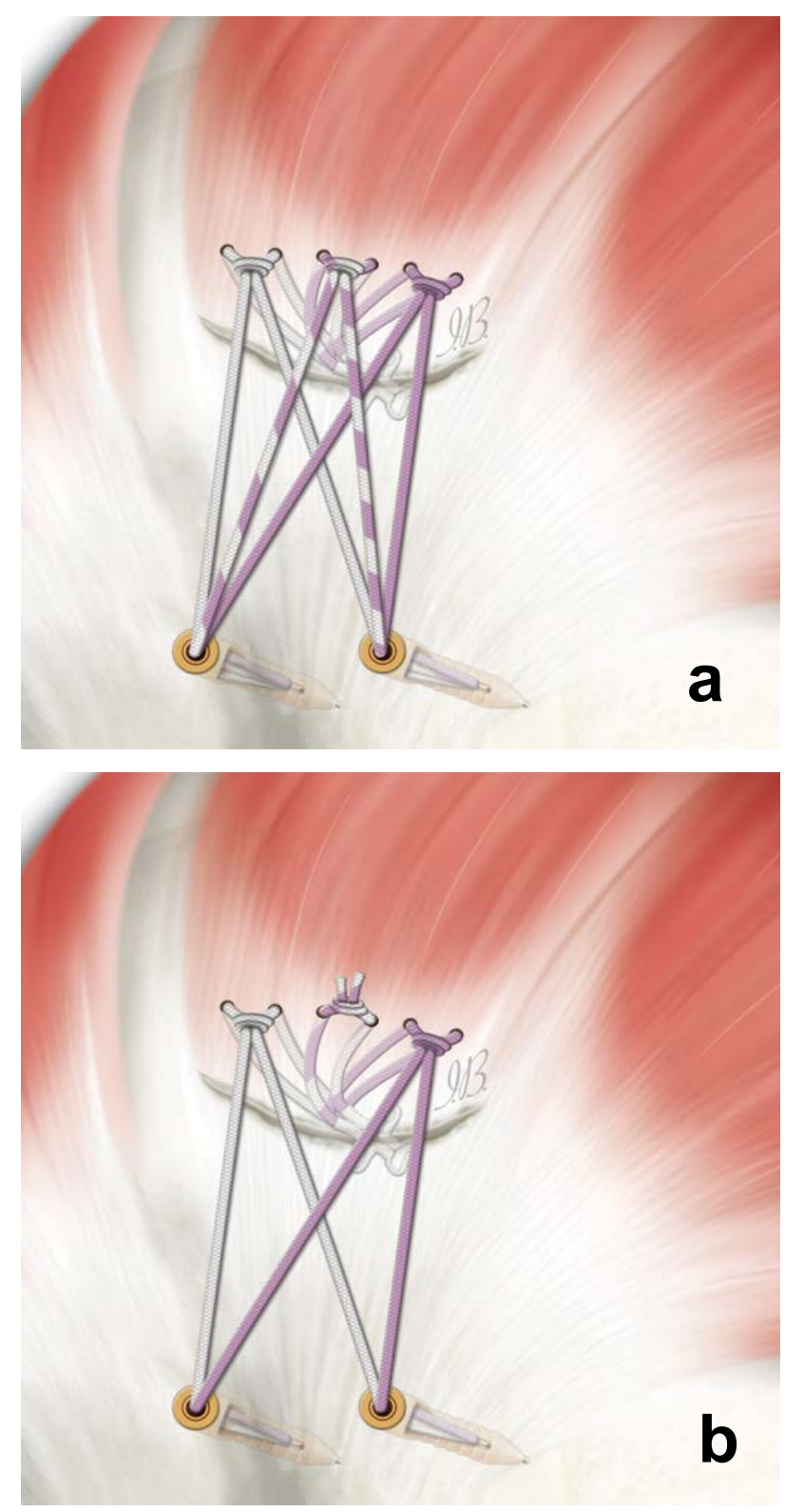

Figure 4: Configuration \#4. double-loaded all-suture anchors (with wires or tape): The sutures are passed through the tendon from posterior to anterior and proceed to close the medial row with mattress knots. When using the anchor with wires the sutures are not cut.

Lateral row: A suture limb from each medial suture anchor is loaded on the knotless anchor which is inserted in the proximal lateral humerus, and then tensioned through the anchor system. The remaining four suture limb, one from each medial anchor, is then fixed with another knotless anchor. Using the construct with the tape, it is not possible to insert all the ends in the eyelet of knotless anchors: After making the knots, a thread is cut for each pair; the remaining tapes are fixed with 2 lateral knotless anchors: By crossing the tapes, a double row X-Box configuration can be obtained (Figure 5a).

\section{Configuration \#5.1}

The terminals of each medial anchor can be loaded inside
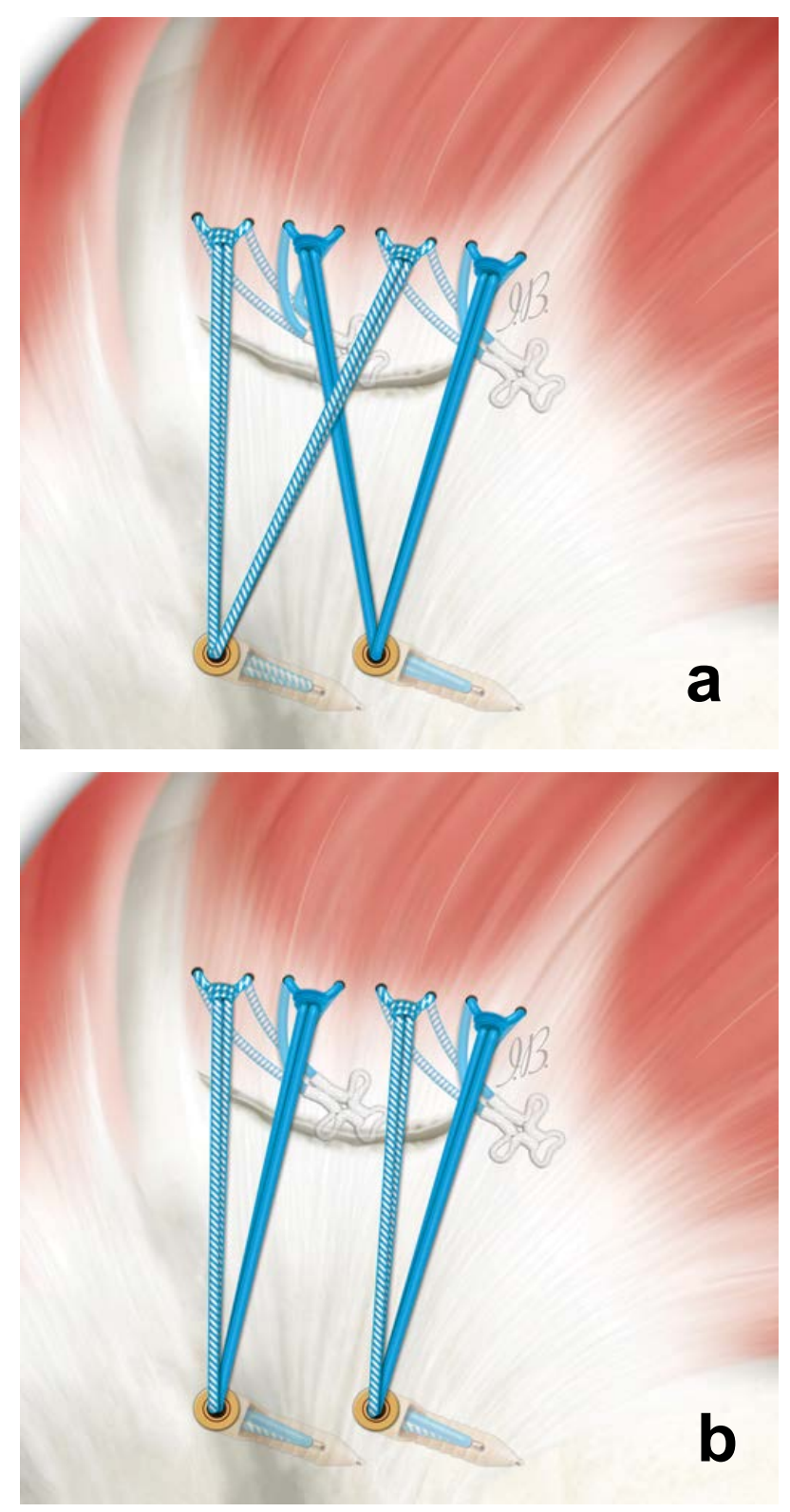

Figure 5: Configuration \#5. 
Citation: Chillemi C, Guerrisi M, Polizzotti G, et al. (2021) Arthroscopic Rotator Cuff Repair: Advancements in Techniques and Technology. Adv Tech Musculoskelet Surg 5(1):59-65

the knotless lateral anchors obtaining an in-line configuration (Figure 5b).

\section{Discussion}

Taking into account the biomechanical data presented in the literature on $\mathrm{RC}$ repair configurations the most interesting aspect of this paper is the development of a repair algorithm that hopefully can help the surgeon to choose the appropriate technique based on RC tear pattern. Obviously, every shoulder surgeon needs to follow the general principles of arthroscopic technique that have been well reported in literature.

It is important in any repair to firstly define the tear pattern. In presence of some kind of tear shapes (such as $U$ - and L-shaped) in order to reduce the size and the tension of the repair construct may be useful to perform margin convergence (i.e. side-to-side), and eventually an anterior and/or posterior interval releases in presence of a retracted tendon [18].

In 2001, Apreleva [19] in a 3D-model study had shown that with the single row repair technique, only $67 \%$ of the original footprint was restored. Based on these results and on the hypothesis that greater coverage of the footprint may promote the repair and the recovery of strength, in 2003 Lo and Burkhart [18] proposed a double row technique in order to increase the footprint and theoretically a greater surface area for tendon-to-bone healing. By adding a second row of anchors, the author speculated that increasing the fixation points increases the strength of the construct; furthermore decreasing the load on each knot reduces the stress at each point of contact of the suture cuff. In a meta-analysis conducted in 2013 by Chen, et al. [20], a greater bone-tendon contact surface emerged compared to the single-row technique.

Ahmad, et al. [21] have noticed significantly more fluid in the healing area when using single-row repair versus a TOE repair, which could prevent healing. Mazzocca, et al. [13] reported that a TOE construct with medial knots had greater pressure and contact area initially and persisted better over time. Consequently, tying of the medial row seems to promote a wider contact surface and reduction of the gap formation with TOE.

Over the years, several studies have compared the single-row technique versus the double-row technique. Charousset [22] in a prospective, nonrandomized study comparing clinical and tendon healing with computed tomographic (CT) orthography found no statistically significant differences regarding the clinical outcome evaluated with Constant score between SR and DR, but a greater tendon healing was registered with orthography in DR group: Aydin, et al. [23] comparing small and medium tear size, did not detect statistically significant difference between single and double-row groups. $\mathrm{Ma}$, et al. [24] on the other hand, also considered patients with different pattern and size of the cuff tear; the author found no difference in clinical scores (UCLA scores, ASES index and muscle strength) but in patients with tear size larger than $3 \mathrm{~cm}$, the double-row group had better shoulder strength in abduction and external rotation than the single-row group, suggesting that this technique be used in these patients; Park, et al. [25] reported similar results, showing that double-row construct yielded better shoulder strength than single-row repair in patients with large to massive lesions $(>3 \mathrm{~cm})$.

Milano [26] in a biomechanical study conducted on fresh pigs shoulders postulated the superiority of the double-row construct from the mechanical point of view that may provide significantly better resistance to elongation and failure at cyclic loading than single-row repair: This can reduce the risk of a gap formation or failure during normal daily activities or as a consequence of a traumatic event, before biological fixation has occurred.

More recently [27] it has been highlighted as the TOE configuration tend to increase traction, converting the negative effects into a compression force that acts perpendicularly to the surface of the greater tuberosity. The increase in the footprint of TOE tendon-bone repairs it could also be more effective in creating a "watertight" repair, which prevents synovial fluid from bathing repair the site in such a way as to compromise the tendon-bone healing [21].

Tudisco, et al. [28] Comparing single-row and double-row re-tear rate, using 3 Tesla MR Imaging, showed a significantly lower re-tear rate in the DR group compared to the SR group ( $25 \%$ vs. $60 \%$. $P=0.02$ ). A meta-analysis conducted by $X u$, et al. [29], which included 9 studies, it emerged that the re-tear rate was higher using the single-row compared to the double-row fixation; The analysis also showed that double-row has a better clinical outcome in larger lesions $(>3 \mathrm{~cm})$, as previously reported. Similar results on the re-rupture emerge from a review in which single-row repairs resulted in a significantly higher re-tear rate compared with double-row repairs, especially with regard to partial-thickness re-tears [30].

A frequently disputed question for the DR repair is if is better to tie or not to tie the medial row. Some authors argue that knots in the medial row have a deleterious effect on repair as excessive pressure in the bone-tendon interface can reduce blood flow deemed important for repair. More recently the use of flattened tape allows a more uniform distribution of forces on the tendon, improve tissue cut-through resistance, enhance self-reinforcement, and improve vascular inflow [9].

A review [31] analyzed the need to knot the medial row rather than using a knotless construct. From the 5 studies included in the analysis it emerge a significant improvement in hysteresis, gap formation, and ultimate load to failure when knots were tied down to the medial-row anchors.

In conclusion, for chronic large degenerative tears a double-row or TOE repair configuration will best achieve the goals of restoring the anatomic footprint, interconnectivity, and mechanical stability to optimize the anatomic healing rates. Medial zing the repair site on the footprint may occasionally be necessary to close the cuff tear: However, biologic healing rates may be lower in such cases.

Basically, anytime may be possible to close the cuff tear 
and completely cover and reattach the footprint without excessive repair tension. In tears in which excessive central cuff crescent tissue erosion or retraction has occurred, the humeral head may not be able to be completely covered, but if the cables (anterior and posterior extent of the tear) can be reattached, the cuff will have improved function. Nowadays, with the advancements in technique and technology is possible to affirm that doesn't exist anymore an irreparable rotator cuff tear, but exist rotator cuff tear that are unable to heal. In the next future the main topic of the research has to be the improvement of the biology.

\section{Acknowledgement}

Special thanks to Miss Ilaria Bondi, medical graphic designer (www.illustrazionianatomiche.com) for preparing all the illustrations of this paper.

\section{References}

1. Chillemi C, Petrozza V, Garro L, et al. (2011) Rotator cuff re-tear or non-healing: Histopathological aspects and predictive factors. Knee Surg Sports Traumatol Arthrosc 19: 1588-1596.

2. Denard PJ, Burkhart SS (2013) The evolution of suture anchors in arthroscopic rotator cuff repair. Arthroscopy 29: 1589-1595.

3. Mantovani M, Pellegrini A, Garofalo P, et al. (2016) A 3D finite element model for geometrical and mechanical comparison of different supraspinatus repair techniques. J Shoulder Elbow Surg 25: 557-563.

4. Castagna, Alessandro, Gumina, et al. (2019) Technique for a novel arthroscopic transosseous rotator cuff repair. Techniques in Shoulder \& Elbow Surgery 20: 12-18.

5. Brian C, El Attrache, Ammar A, et al. (2007) Arthroscopic rotator cuff repairs: An anatomic and biomechanical rationale for different suture-anchor repair configurations. Arthroscopy 23: 662-669.

6. Caldwell GL, Warner JP, Miller MD, et al. (1997) Strength of fixation with transosseous sutures in rotator cuff repair. J Bone Joint Surg 79: 1064-1068.

7. Van der Zwaal P, Thomassen BJ, Nieuwenhuijse MJ, et al. (2012) clinical outcome in all arthroscopic versus mini-open rotator cuff repair in small to medium-sized tears: A randomized controlled trial in 100 patients with 1-year follow-up. Arthroscopy 29: 266273.

8. Park MC, Elattrache NS, Ahmad CS, et al. (2006) Transosseous-equivalent rotator cuff repair technique. Arthrosc J Arthrosc Relat Surg 22:1360-1365.

9. Liu RW, Lam PH, Shepherd HM, et al. (2007) Tape versus suture in arthroscopic rotator cuff repair: Biomechanical analysis and assessment of failure rates at 6 months. Orthop J Sports Med 5: 2325967117701212.

10. Chillemi C, Castagna A, Osimani M, et al. (2018) Rotator cuff tear: Imaging. In: Arthroscopic transosseous rotator cuff repair: Tips and tricks. Springer, 23-29.

11. Chillemi C, Castagna A, Osimani M, et al. (2018) Rotator cuff tear: Classification system. In: Arthroscopic transosseous rotator cuff repair: Tips and tricks. Springer, 11-21.

12. Snyder SJ (2002) Arthroscopic classification of rotator cuff lesions and surgical decision making. In: Shoulder arthroscopy. Philadelphia: Lippincott William \& Wilkins, 201-202.

13. Mazzocca AD, Chowaniec D, Cote MP, et al. (2012) Biomechanical evaluation of classic solid and novel all-soft suture anchors for glenoid labral repair. Arthroscopy 28: 642-648.
14. Alemanno F, Gretter R, Di Leo Y, et al. (2003) Alemanno's brachial plexus block ten years later: Topographic study of the anesthetized areas. Minerva Anestesiol 69: 575-581.

15. Snyder S, Burns J (2009) Rotator Cuff Healing and the Bone Marrow "Crimson Duvet" From Clinical Observations to Science. Techniques in Shoulder \& Elbow Surgery 10: 130-137.

16. Gigante A, Marinelli M, Chillemi C, et al. (2004) Fibrous cartilage in the rotator cuff: A pathogenetic mechanism of tendon tear?. J Shoulder Elbow Surg 13: 328-332.

17. Zumstein MA, Raniga S, Labrinidis A, et al. (2016) Optimal lateral row anchor positioning in posterior-superior transosseous equivalent rotator cuff repair: A micro-computed tomography study. Orthop J Sports Med 22: 4.

18. Lo IK, Burkhart SS (2003) Double-row arthroscopic rotator cuff repair: Re-establishing the footprint of the rotator cuff. Arthroscopy 19: 1035-1042.

19. Apreleva M, Ozbaydar M, Fitzgibbons PG, et al. (2002) Rotator cuff tears: The effect of the reconstruction method on three dimensional repair site area. Arthroscopy 18: 519-526.

20. Chen $M, X u W$, Dong $Q$, et al. (2013) Outcomes of single-row versus double-row arthroscopic rotator cuff repair: A systematic review and meta-analysis of current evidence. Arthroscopy 29: 1437-1449.

21. Ahmad CS, Vorys GC, Covey A, et al. (2009) Rotator cuff repair fluid extravasation characteristics are influenced by repair technique. J Shoulder Elbow Surg 18: 976-981.

22. Charousset C, Grimberg J, Duranthon LD, et al. (2007) Can a double-row anchorage technique improve tendon healing in arthroscopic rotator cuff repair?: A prospective, nonrandomized, comparative study of double-row and single-row anchorage techniques with computed tomographic arthrography tendon healing assessment. Am J Sports Med 35: 1247-1253.

23. Aydin N, Kocaoglu B, Guven O, et al. (2010) Single-row versus double row arthroscopic rotator cuff repair in small- to medium-sized tears. J Shoulder Elbow Surg 19: 722-725.

24. Ma HL, Chiang ER, Wu HT, et al. (2012) Clinical outcome and imaging of arthroscopic single-row and double-row rotator cuff repair: A prospective randomized trial. Arthroscopy 28: 16-24.

25. Park JY, Lhee SH, Choi JH, et al. (2008) Comparison of the clinical outcomes of single- and double-row repairs in rotator cuff tears. Am J Sports Med 36: 1310-1316.

26. Milano G, Grasso A, Zarelli D, et al. (2008) Comparison between single-row and double-row rotator cuff repair: A biomechanical study. Knee Surg Sports Traumatol Arthrosc 16: 75-80.

27. Andrew S, Robert H, Burkhart S, et al. (2019) Arthroscopic rotator cuff repair in 2019: linked, double row repair for achieving higher healing rates and optimal clinical outcomes. Arthroscopy 35: 2749-2755.

28. Tudisco C, Bisicchia S, Savarese E, et al. (2013) Single-row vs. double-row arthroscopic rotator cuff repair: Clinical and 3 tesla $\mathrm{mr}$ arthrography results. BMC Musculoskelet Disord 14: 43.

29. Xu C, Zhao J, Li D, et al. (2014) Meta-analysis comparing single-row andvdouble-row repair techniques in the arthroscopic treatment of rotator cuff tears. J Shoulder Elbow Surg 23: 182-188.

30. Millett PJ, Warth RJ, Dornan GJ, et al. (2014) Clinical and structural outcomes after arthroscopic single-row versus double- row rotator cuff repair: A systematic review and meta-analysis of level I randomized clinical trials. J Shoulder Elbow Surg 23: 586-597.

31. Mall NA, Lee AS, Chahal J, et al. (2013) Transosseous-equivalent rotator cuff repair: A systematic review on the biomechanical importance of tying the medial row. Arthroscopy 29: 377-386. 